\title{
Spectroscopic identification of Quinacridone polymorphs for organic electronics†
}

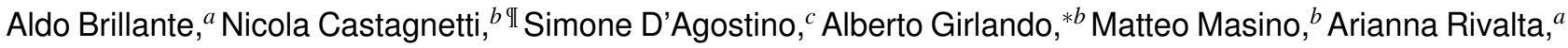 \\ Tommaso Salzillo, ${ }^{a \ddagger}$ and Elisabetta Venuti ${ }^{a}$
}

Quinacridone is a well known dye, recently proposed as an environmentally friendly, air stable organic semiconductor. The polymorphism of Quinacridone has been widely debated, since dif-ferent polymorphs exhibit different colors, but the problem has not been addressed in relationship with its use in organic electronics. Here we give a detailed account of how the combination of different nondestructive spectroscopic techniques (Raman, infrared, luminescence), also applicable to a working device, can be used to identify the Quinacridone polymorphs. This is the necessary starting point to optimize the growth conditions towards the polymorph with the best performance for the sought application.

\section{Introduction}

In a few years organic electronics has evolved in a multi-billion dollar industry, mainly due to the breakthrough of OLED displays. Yet, several of its promises, such as long-term stability coupled to bio-compatibility, have still to be fulfilled. From this perspective, the proposal of using commercial or natural dyes as organic semiconductors appeared quite appealing. $1+3$ In these systems, despite the perspective of using hydrogen bonding and the methods of crystal engineering 4 to build the most suitable (and airstable) crystalline phase, the problem of polymorphism ${ }^{5}$ remains one of the issues still to be fully investigated and overcome.

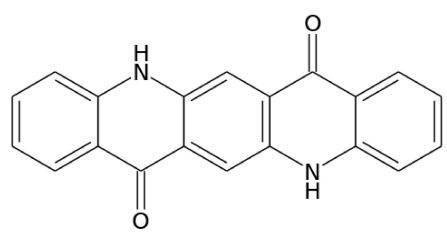

Fig. 1 Quidacridone chemical structure.

\footnotetext{
$\uparrow$ Electronic Supplementary Information (ESI) available: [details of any supplementary information available should be included here]. See DOI: 10.1039/b000000x/ a Dipartimento di Chimica Industriale “Toso Montanari” and INSTM-UdR Bologna, Università of Bologna, Viale del Risorgimento 4, 40136 Bologna, Italy

${ }^{b}$ Dipartimento di Scienze Chimiche, della Vita e della Sostenibilità Ambientale and INSTM-UdR Parma, Università di Parma, Parco Area delle Scienze, 43124 Parma, Italy. c Dipartimento di Chimica "Giacomo Ciamician", Università of Bologna, Via Selmi 2, 40126 Bologna, Italy.

ๆ Present address: Piacenza

* Present address: Present address: Institut de Ciéncia de Materials de Barcelona (CSIC), Campus de la UAB, 08193 Bellaterra (Spain).Barcelona
}

In this paper we address the question of polymorphism in Quinacridone (5,12-Dihydroquinolino[2,3-b] acridine-7,14dione, henceforth abbreviates as QA, Figure 1). QA is a low-cost commercial dye widely used in paints and colors industry, and as such has been subjected to many investigations, both structural and spectroscopic, 67 since different polymorphs display different colors. However, considerable confusion was present in the literature, due to patent issues and to a different nomenclature which in case of dyes refer directly to color, so that QA is named PV-19 (Pigment Violet 19), without reference to different phases. At the same time the generic name "Quinacridone" has been used for both the actual QA or for other chemically different derivatives, e.g. 2,9-dimethyl-QA. ${ }^{8}$ Furthermore, chemical impurities or controlled doping may also be used to affect the color.

Thanks to a series of accurate structural studies, it is now accepted that pure QA can be obtained in four different forms, named $\alpha^{\mathrm{I}}, \alpha^{\mathrm{II}}, \beta$ and $\gamma \cdot{ }^{910}$ The first two polymorphs can only be obtained as powders, are affected by considerable disorder and are not as stable as the other two. Hence, they are not used as pigment, and are of no relevance here, except for their possible presence in the stable, crystalline phases of $\beta-$ and $\gamma-\mathrm{QA}$. We have already shown how Raman spectroscopy in the lattice phonons region (10-150 $\mathrm{cm}^{-1}$ ) can be used, also directly on the device, to identify organic semiconductor polymorphs. 11 Here, we also adopt other spectroscopic techniques, since in the case of QA Raman can be hampered by strong luminescence, a feature that can be profitably used in QA-based photovoltaic devices. 12 


\section{Experimental}

\subsection{Samples preparation}

Powders of the $\alpha^{\mathrm{I}}$ phase were obtained according to the reported procedure. ${ }^{13}$ Powderss (100 mg) of commercial QA from SigmaAldrich were dissolved in $96 \%$ sulfuric acid $(10 \mathrm{~mL})$ sonicated for $1 \mathrm{~h}$, filtered, and kept overnight stirring at room temperature. Powders of $\alpha^{\mathrm{I}}-\mathrm{QA}$ were obtained by adding drop wise the resulting solution to a beaker filled up with ice. The precipitated material was filtered and washed with abundant water (50 x 10 $\mathrm{mL}$ ). Powders of the $\beta$ and $\gamma$ phases, prepared as described by Paulus et al., 9 were kindly provided by M. Schmidt.

Single crystals were obtained by the physical vapor transport (PVT) method, starting from commercial powder (TCI, purity > 93.0\%). Crystals subsequently characterized as $\beta-\mathrm{QA}$ were obtained by inserting the powders in a glass tube about $10 \mathrm{~cm}$ long, sealed under low vacuum $\left(\approx 10^{-3}\right.$ mbar $)$. The sealed tube was inserted in a furnace with a linear temperature profile, the extremes being set at $340{ }^{\circ} \mathrm{C}$ (sublimation zone) and $300{ }^{\circ} \mathrm{C}$ (deposition area). The growth was stopped after 6 days. Single crystals of the $\gamma$ - phase was instead obtained by the open-tube PVT method, 14 where a controlled constant flux of Nitrogen was applied during the growth $(80 \mathrm{~h})$. The sublimation temperature at one end of the tube was $300^{\circ} \mathrm{C}$, and the estimated temperature in the crystallization zone was $\approx 250-200{ }^{\circ} \mathrm{C}$. Pictures of the crystalline samples are reported in Fig. 2. Crystals of $\beta-\mathrm{QA}$ are thicker than those of $\gamma-\mathrm{QA}$, so that images in reflected light better show the change of color observed by polarizing the light along the different extinction directions (indicated in the Figure).

QA films on glass and $\mathrm{KBr}$ plates were prepared with in-house modified vacuum metal coater, under the high vacuum $\left(\approx 10^{-7}\right.$ mbar) of a diffusion pump. The temperature of the furnace, a glass basket loaded with QA, was between 350 and $400 \mathrm{~K}$, and the substrate was suspended about $3 \mathrm{~cm}$ above the furnace. No cooling or temperature control of the substrate was available, nor a way of controlling the amount of deposited material. Different films were prepared just by changing the exposure time.
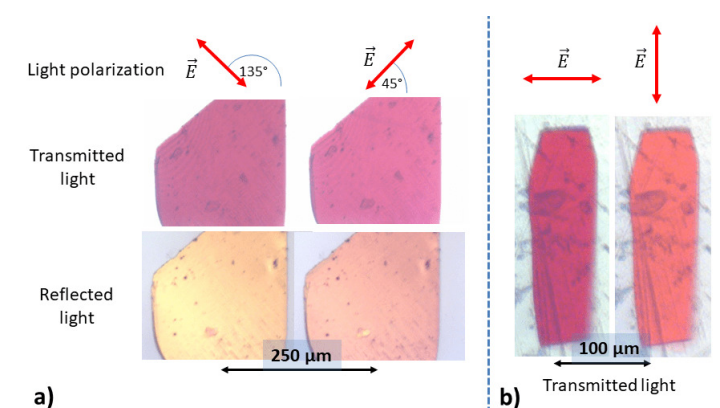

Fig. 2 Left side: $\beta-Q A$ crystal (face not identified) grown by closed-tube PVT, viewed in transmission and reflection, with light polarized along the indicated extinction directions. Right side: $\gamma-\mathrm{QA}$ crystal (likely the $b c$ face) grown by open-tube PVT, viewed in transmission with light polarized along the indicated extinction directions.

\subsection{Spectral measurements}

The Raman spectra were collected both by a JY-Horiba T64000 triple Raman spectrometer interfaced to a Olympus BX40 microscope and by Renishaw 1000 spectrometer coupled to a Leica M microscope. Excitation at 476, 647 and $752 \mathrm{~nm}$ was provided by a $\mathrm{Kr}$ ion laser. The polarization of the incident laser radiation was controlled by a half-wave polarization rotator, and the scattered light was analyzed by a rotating polaroid polarizer.

Infrared (IR) spectra were recorded with a Bruker IFS66 FTIR spectrometer, coupled to an Hyperion 1000 IR microscope equipped with a liquid Nitrogen cooled mercury cadmium telluride (MCT) detector. Polarized light was obtained by a rotating wire grid polarizer.

Luminescence spectra of the QA powder and films were recorder with an Edimbourgh Instruments FLS 1000 spectrometer.

\subsection{X-ray measurements}

$\mathrm{X}$-ray powder diffractograms in the $2 \theta$ range 5 -401 (step size, 0.021; time per step, $20 \mathrm{~s}$; 0.04 rad soller; $40 \mathrm{~mA} \mathrm{x} 40 \mathrm{kV}$ ) were collected on a PANalytical X'Pert PRO automated diffractometer equipped with an X'Celerator detector and in Bragg-Brentano geometry, using $\mathrm{CuK} \alpha$ radiation without a monochromator. Identity between bulk materials and crystal structures was verified by comparing experimental and calculated powder diffraction patterns (see Electronic Supplementary Information, ESI).

\section{Reference spectra}

\subsection{Single crystal Raman and IR spectra}

It is well known that Raman spectroscopy, notable in the region of lattice vibrations, is a powerful tool for identification of polymorphs.11|15|16 The case of QA presents some difficulties, due to the interfering effect of luminescence, which incidentally is also different for different QA polymorphs (see below). The problem is marginal for red excitation (647 and $752 \mathrm{~nm}$ ) in the case of single crystals in the low-frequency region (below $200 \mathrm{~cm}^{-1}$ ), albeit some baseline correction has to be applied in some cases. Heavy baseline correction is needed for the ordinary spectral region 400 $-3500 \mathrm{~cm}^{-1}$, either with the use of red or blue $(476 \mathrm{~nm})$ excitation. We report the relevant spectra in the ESI and we shall not discussed any further here, as they are of limited use for the aim of the present paper.

The polarized low frequency Raman spectra of $\beta-$ and $\gamma-\mathrm{QA}$ single crystals are reported in Fig. 3. Both polymorphs crystallize in the monoclinic system, $P 2_{1} / c\left(C_{2 h}^{5}\right)$ with two molecules per unit cell, 9 so that in the rigid molecule approximation one expects 6 Raman active lattice vibrations, 3 of $A_{g}$ and 3 of $B_{g}$ symmetry. 17 The $A_{g}$ modes are visible in the $(a a),(b b),(c c)$ and $(a c)$ polarization, the $B_{g}$ ones in the remaining $(a b)$ and $(b c)$ polarization.

Figure 3 shows that indeed six Raman bands are observed in the low frequency region of both polymorphs (the frequencies are reported in the Figure). The crystals of $\beta-\mathrm{QA}$ are rather irregular in shape (the one in Figure 2 is a rare exception), and the crystal faces likely are not principal faces. The polarized spectra therefore do not allow to identify the symmetry species, the 


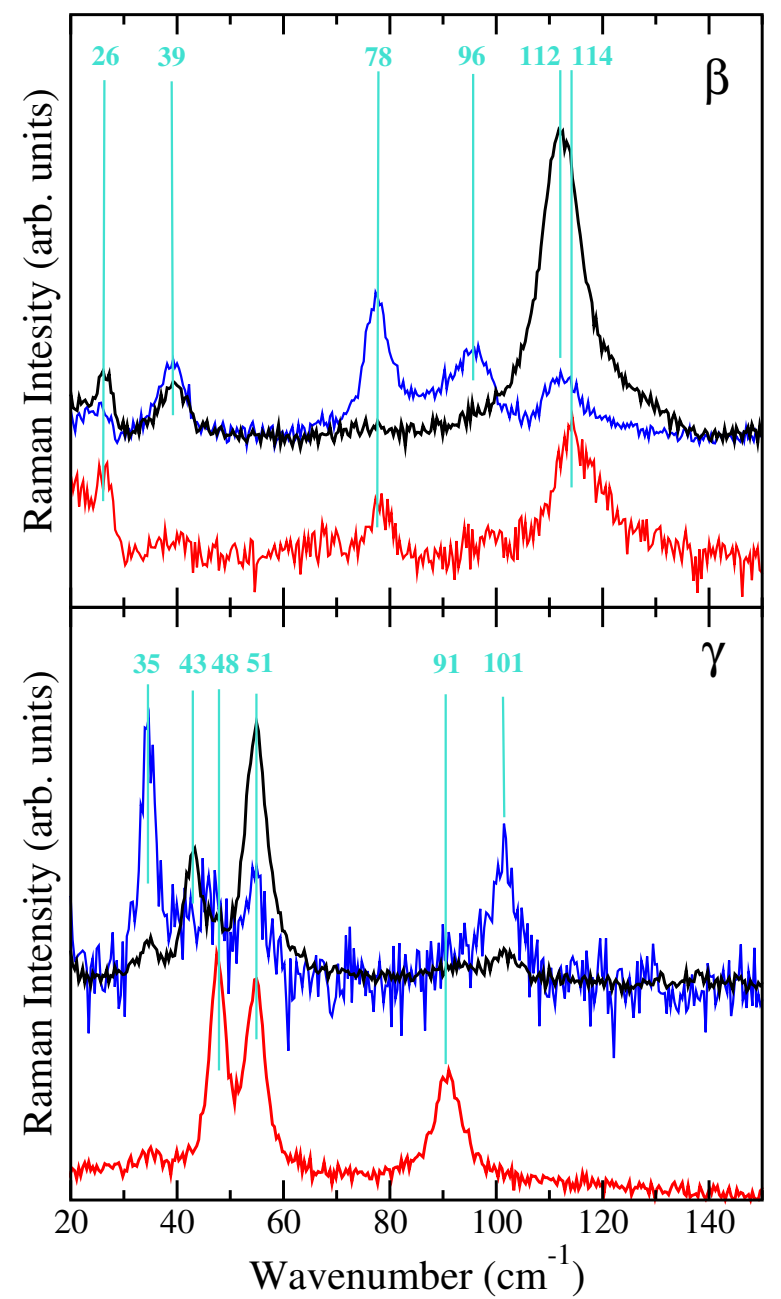

Fig. 3 Polarized low-frequency Raman spectra of $\beta$ - (top panel) and $\gamma-\mathrm{QA}$ (bottom panel) single crystals (exciting line: $647 \mathrm{~nm}$ ). The spectra are offset for clarity. The black and blue line spectra have been collected with both polarizer and analyzer directed along the extinction direction, the red line one has been recorder with crossed polarization.

bands occurring at the same frequency (but different intensity) in the different polarizations. On the contrary, $\gamma-\mathrm{QA}$ often presents elongated crystal face identified as the $b c$ [100] one, so that the $B_{g}$ modes are expected (and found) in the crossed polarizer/analyzer spectra only (bottom panel of Fig. 33). Discussing the assignment and mode description goes beyond the scope of this paper. A look at the Figure, on the other hand, shows that the spectral pattern is clearly different for the two polymorphs. We shall discuss more in detail the polymorph discrimination through Raman in the next Subsection, where we will present the powders spectra.

Mid-IR spectroscopy has been already proposed as a method to distinguish QA polymorphs. ${ }^{6}$ In particular the $\beta$ - and $\gamma$ - polymorphs can be distinguished by looking at the $\mathrm{CH}$ stretching region. $\frac{18}{}$ Here we present the polarized single crystal IR absorption spectra, which allow to better appreciate the origin of the difference. The spectra obtained from the samples shown in Fig. 2 are shown in Fig. 4 Apart from the differences in the band shape (evident in the $\mathrm{CH}$ stretching region) the spectra of $\gamma-\mathrm{QA}$ appear very strongly polarized, whereas the difference between the two

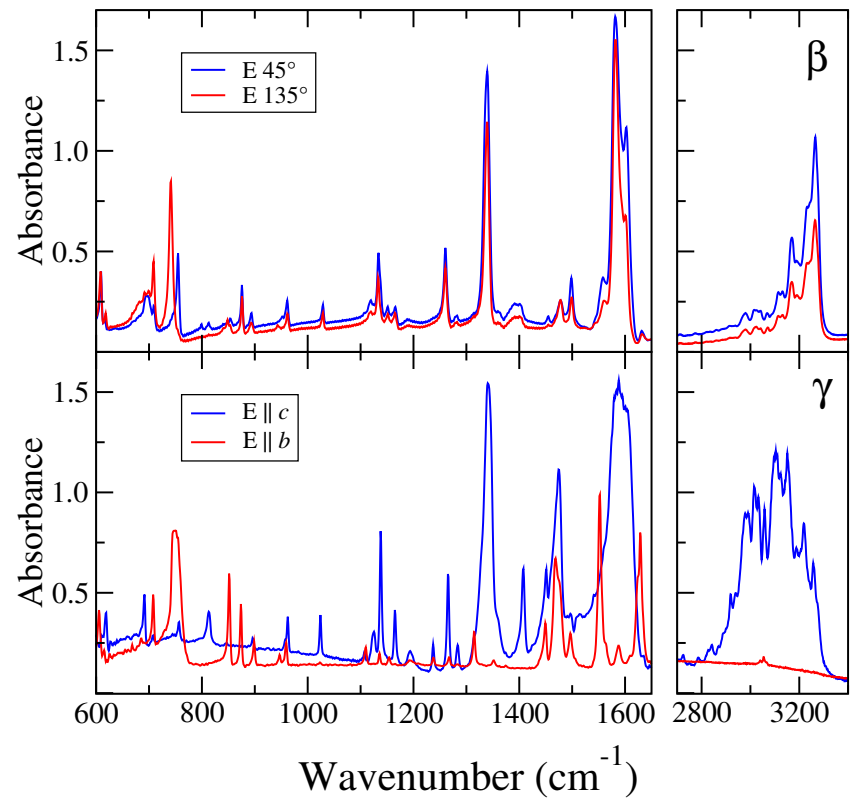

Fig. 4 Polarized IR spectra of $\beta$ - and $\gamma-\mathrm{QA}$. The samples are those reported in Fig. 2 with the indication of the extinction directions.

polarization is far less marked in $\beta-\mathrm{QA}$.

The difference in the spectra can be easily understood by observing the crystalline structures of $\beta-\mathrm{QA}$ and $\gamma-\mathrm{QA}^{9}$ (CCDC: $620258,620259)$ and reported in Fig. 5, that evidence the different $\mathrm{H}$-bond frameworks of the two polymorphs. In $\beta-\mathrm{QA}$ each molecule is bonded trough two H-bonds to the nearest two neighboring molecules, forming two non-parallel chains, one half running in the [110] direction, and the other half in the [110] direction. As a consequence H-bonds develop perpendicularly to these two directions, so that the bands are not polarized. In $\gamma-\mathrm{QA}$ on the contrary each molecule is connected by single hydrogen bonds to four neighboring molecules, so that the molecules are not arranged in chains, but form a criss-cross pattern, and H-bond network develops along a unique direction. ${ }^{9}$ The same considerations apply to the vibrations whose oscillating dipole moment is directed along the in-plane short axis of QA molecule, like for instance the $\mathrm{C}=\mathrm{O}$ stretching vibration around $1600 \mathrm{~cm}^{-1}$.

\subsection{Spectroscopic characterization of QA's powders}

The single crystal Raman and IR spectra allow easy discrimination of $\beta-$ and $\gamma-\mathrm{QA}$ polymorphs. However, polycrystalline or amorphous thin films are used in devices, and these may present mixture of the phases, or the presence of the $\alpha^{\mathrm{I}}$ polymorph, which is not known as single crystal. ${ }^{9}$ Therefore before proceeding to the characterization of some thin films, we shall present reference spectra for bulk powdered samples.

Fig. 6 presents the low-frequency Raman spectra of $\alpha^{\mathrm{I}}-, \beta-$ and $\gamma-\mathrm{QA}$ powders. For comparison we report as dotted lines the spectra of $\beta$ - and $\gamma-\mathrm{QA}$ single crystals from Fig. 3 , averaged over the different polarizations. Furthermore, the spectral region is extended towards higher frequencies in order to show another pair of bands, between 200 and $250 \mathrm{~cm}^{-1}$. These latter bands have mostly intra-molecular character, since they are broadened 

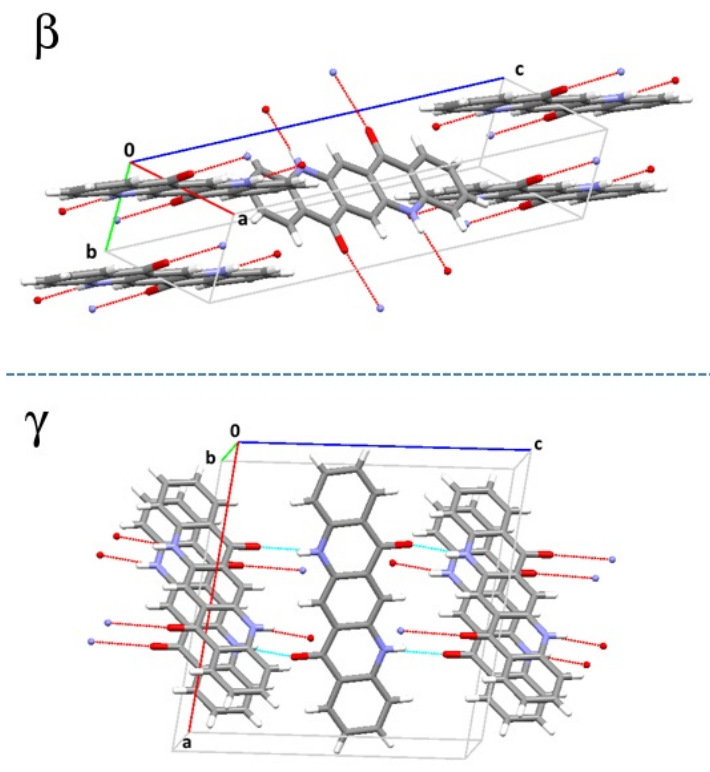

Fig. 5 X-ray crystal structure of $\beta-\mathrm{QA}$ (top) and $\gamma-\mathrm{QA}$ (bottom).

just a little in the powders with respect to the crystal: We expect intra-molecular modes to be almost dispersionless, so that disorder does not affect strongly their bandwidth. Yet, we observe that the band at $216 \mathrm{~cm}^{-1}$ is much broader in $\alpha^{\mathrm{I}}-\mathrm{QA}$ than in $\beta-\mathrm{QA}$ (it is not detected in $\gamma-\mathrm{QA}$ ), and that the band around $230 \mathrm{~cm}^{-1}$ occurs at higher frequency in $\gamma-\mathrm{QA}$ with respect to the other two polymorphs.

It is not surprise that the Raman spectrum of $\alpha^{\mathrm{I}}-\mathrm{QA}$ is rather similar to that of $\beta-\mathrm{QA}$, since the two structures are similar, with the same hydrogen bonding pattern, albeit in $\alpha^{\mathrm{I}}-\mathrm{QA}$, with one QA molecule per unit cell, all the hydrogen bonded chains are equally oriented. ${ }^{9}$ In $\alpha^{\mathrm{I}}-\mathrm{QA}$ we expect three Raman active lattice phonons. Just one broad band is clearly identified around 97 $\mathrm{cm}^{-1}$, and its frequency is appreciably different from the prominent features of $\beta$ - and $\gamma-\mathrm{QA}$ in the same spectral region. Other frequency differences are evidenced directly in the Figure. As a consequence, all the three QA polymorphs can be discriminated by their low-frequency Raman spectra.

Fig. 7 shows the IR spectra of perfluorocarbon mulls of $\alpha^{\mathrm{I}}-$, $\beta-$ and $\gamma-\mathrm{QA}$. As already noted in the case of the crystals, the $\mathrm{CH}$ stretching region (right panel of the Figure) allows easy discrimination of $\beta-$ and $\gamma-\mathrm{QA}$. However, in this spectral region $\alpha^{\mathrm{I}}-\mathrm{QA}$ has just a broader pattern with respect to the $\beta$ - polymorph. A careful analysis of the $1300-1650 \mathrm{~cm}^{-1}$ spectral region is required to identify small but significant frequency differences between the three polymorphs, as evidenced by the vertical lines and frequencies reported in the left panel of Fig. 7. Therefore also the IR spectra can be used to discriminate between $\alpha^{\mathrm{I}}-, \beta-$ and $\gamma$ - polymorphs.

In view of the color difference, the electronic absorption spectra would seem the most direct method to identify the QA polymorphs. However, obtaining the relevant reference spectra is very problematic: As a matter of fact, our crystalline samples yield in

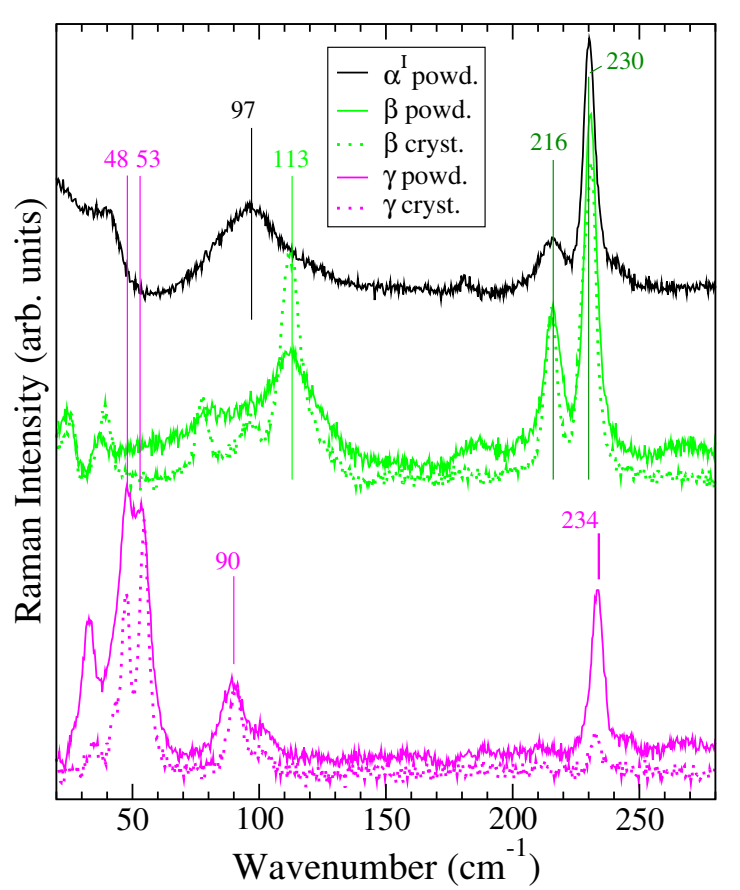

Fig. 6 Raman spectra of $\alpha^{\mathrm{I}}-, \beta$ - and $\gamma-\mathrm{QA}$ powders in the lowfrequency spectral region. The averaged spectra of $\beta$ - and $\gamma-\mathrm{QA}$ crystals from Fig. 3 are reported for comparison (dotted lines). The vertical lines evidence the main frequency differences between the spectra.

any case saturation, in view of the huge intensity of the transition, and the powders give too much scattering. Attempts to grind the powders to reduce particle size resulted in change of the color following the transformation of all samples into the $\beta$-form. Also the available literature is not very clear in associating a given spectrum with a give polymorph, the conditions of the spectral collection or sample from are not given, and there is no agreement between different papers. $\frac{4 \sqrt{6}}{}$ However, in recording the Raman spectra we realized that the luminescence spectra of the three QA polymorphs were different. Luminescence have the advantage of sensibility and the possibility of being collected directly on the operational device On the other hand, it may be

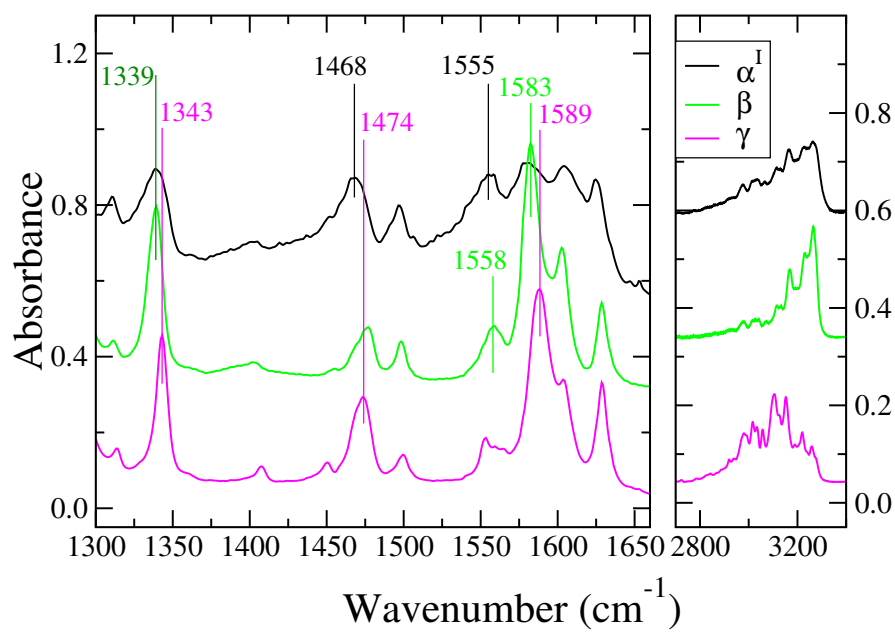

Fig. 7 IR spectra of $\alpha^{\mathrm{I}}-, \beta-$ and $\gamma-\mathrm{QA}$ powders. 


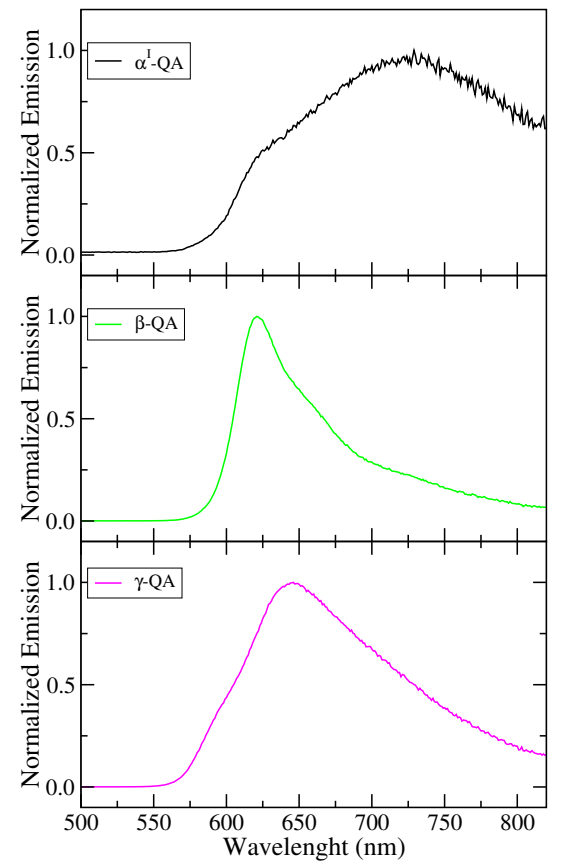

Fig. 8 Emission spectra of $\alpha^{\mathrm{I}}-, \beta$ - and $\gamma-\mathrm{QA}$ powders.

affected by impurities, self-absorption and scattering. We decided in any case to test extensively the luminescence on different samples, and we verified that although there may be difference in the frequencies of the peak maxima, the overall band-shape is reproducible irrespective of the sample form (powders, crystals or polycrystalline samples).

Fig. 8 exemplifies the differences of the luminescence spectra of the powders of the three QA polymorphs: The $\alpha^{\mathrm{I}}$ form display a very broad luminescence, extending from about 600 to over $800 \mathrm{~nm}$, with a hint of a a shoulder around $620 \mathrm{~nm} ; \beta-\mathrm{QA}$ has a neat luminescence spectrum, with the main peak around $620 \mathrm{~nm}$, and evidences of the relevant vibronic progression; finally, $\gamma-\mathrm{QA}$ luminescence has a strange shape, with a shoulder around 600 $\mathrm{nm}$, the main peak around $650 \mathrm{~nm}$, with a slow and structureless decay down to $800 \mathrm{~nm}$.

Investigating the photophysics of the QA luminescence is certainly a complex but worthwhile, task, also in view of the possible application in photovoltaics. ${ }^{12}$ For the aim of the present paper such investigation is not needed, and we limit ourselves to indicate that luminescence can be conveniently used as an alternative or as a complement to Raman and IR to identify QA polymorphs in thin films grown under different conditions, as we illustrate in the next Section.

\section{Polymorph identification in sublimed films}

As explained in Section 2.1. we had not to much control on the growth conditions of the films on substrate. Here we present the results of our spectroscopic investigation on a couple of films grown on a glass slide (film labeled \#2 and \#4), and a couple grown on a $\mathrm{KBr}$ window. The latter are labeled as thin and thick film, in correspondence with the exposure time and optical den-

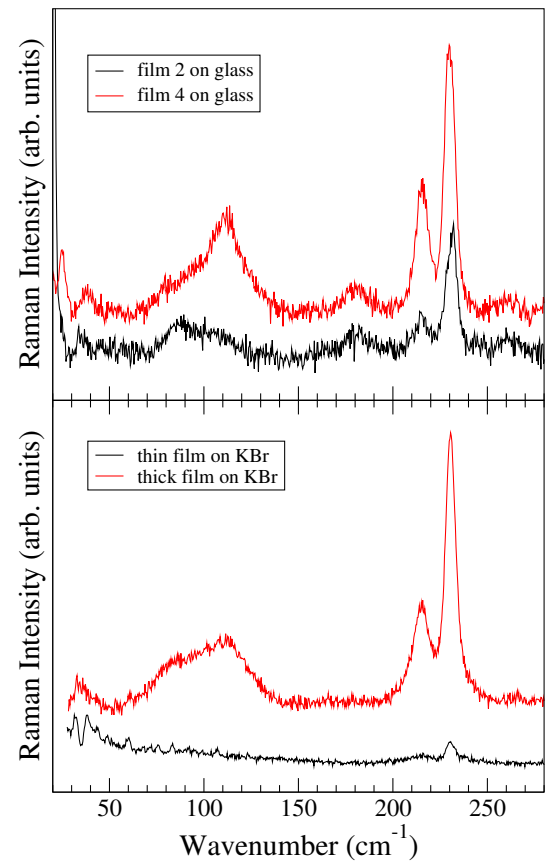

Fig. 9 Low-frequency Raman spectra of $\mathrm{QA}$ films on glass and on $\mathrm{KBr}$ substrate.

sity.

We first show Raman spectra in the low-frequency region, as this is the most specific and reliable method to identify polymorphs in molecular crystals. ${ }^{11}$ The spectra presented here illustrate some difficulties and limitations of the method. First of all, the films have stronger luminescence and scattering than the crystals, so that rather heavy background subtraction has to be performed to extract the Raman bands. In the ESI we report the original spectra, to be compared with the background subtracted spectra in Fig. 9. Second, the presence of disorder in the films considerably broadens the bands related to the lattice modes, as already shown by comparing powders and single crystal spectra in Fig. 6 Finally, in the films we may have the coexistence of different polymorphs at the sub-micrometer scale.

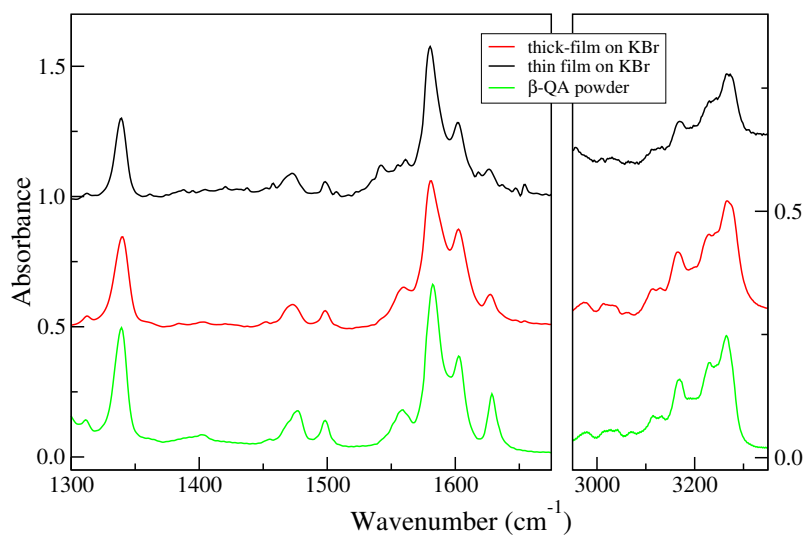

Fig. $10 \mathrm{IR}$ spectra of $\mathrm{QA}$ films on $\mathrm{KBr}$. The spectrum of the $\beta$ polymorph powders (from Fig. 7 is reported for comparison.

The comparison between Fig. 9 and Fig. 6, clearly shows that 
film \#4 on glass (red trace in the top panel) corresponds to the $\beta$ form, as indicated by the broad but still indicative band around $113 \mathrm{~cm}^{-1}$ and by the pair at 216 and $230 \mathrm{~cm}^{-1}$. On the other hand, the spectrum of film \#2 on glass suggests that the films is prevailing made by the $\gamma$ polymorph, as the bands around 113 and $216 \mathrm{~cm}^{-1}$ are strongly weakened. Furthermore, the peak of the most intense band occurs at $233 \mathrm{~cm}^{-1}$, as in the $\gamma$ phase, with possibly a weaker shoulder around $229 \mathrm{~cm}^{-1}$, diagnostic of the $\beta$. The prevailing presence of the $\gamma$ phase is also suggested by a broad band close to $90 \mathrm{~cm}^{-1}$, whereas the absence of the $\gamma$ characteristic doublet around $50 \mathrm{~cm}^{-1}$ can be explained as a large broadening or polarization effect (cf. the blue trace in the bottom panel of Fig. 3).

The bottom panel of Fig. 9 clearly shows that the thick film on $\mathrm{KBr}$ (red trace) belongs to the $\beta$ phase, with possibly a small contamination of $\gamma$-QA. On the other hand, the Raman spectrum of the thin film on $\mathrm{KBr}$ (black trace in the bottom panel) is too weak to be disentangled from the strong fluorescence background, hence cannot be used for polymorph identification.

The IR absorption spectra obviously cannot be collected for samples on glass substrate. The ones for thin and thick film on $\mathrm{KBr}$ are shown in Fig. 10 The comparison with IR spectrum of $\beta-\mathrm{QA}$ (green line in Fig. 8, reported for ease of comparison also at the bottom of Fig. 10 shows that indeed both films grown on $\mathrm{KBr}$ do belong to this form. Contamination by different polymorphs, as hinted from the Raman spectra of the thick film, cannot be confirmed by the IR spectra.

Finally, Fig. 11 reports the luminescence spectra of the QA films on glass (top panel) and on $\mathrm{KBr}$ (bottom panel). Comparison with Fig. 8 confirm that both the films grown on $\mathrm{KBr}$ belong to the $\beta$ polymorph. However, as suggested from the Raman spectra above, the thick film also contains some $\gamma-\mathrm{QA}$, as evidenced from the shoulder around $670 \mathrm{~nm}$, not present in the thin film. On the other hand, the two films grown on glass belong to two different polymorphs, film \#2 to $\gamma-\mathrm{QA}$, and film \#4 to $\beta-\mathrm{QA}$, again confirming the Raman indications. Hence subtle changes of the sublimation conditions can preferentially drive the growth towards one or the other polymorph. No clear evidences of contamination by the $\alpha$ form have been found, although of course this cannot be completely excluded from the present data.

\section{Conclusions}

In this paper we have shown how fast, nondestructive spectroscopic techniques can be used to characterize polymorphism in QA crystals and crystalline thin films for use in organic electronics. In the recent literature, ${ }^{2 \mid 12}$ the QA films grown by sublimation are reported to belong to the $\beta$ phase. However, older literature reported the "exclusive" growth of the $\gamma$ polymorph, which was believed to be the "most heat-stable form".1920 From our limited experience, we think that indeed $\beta-\mathrm{QA}$ is the most stable form at ambient conditions, as hinted also by the fact that simple grinding transform both $\alpha^{\mathrm{I}}-$ and $\gamma-\mathrm{QA}$ transform to the $\beta$-polymorph. On the other hand, we believe that $\gamma$-QA has similar cohesive energy, hence small changes in the sublimation conditions can induce its preferential growth. Simultaneous growth of both polymorphs in the same film is also possible, as in the

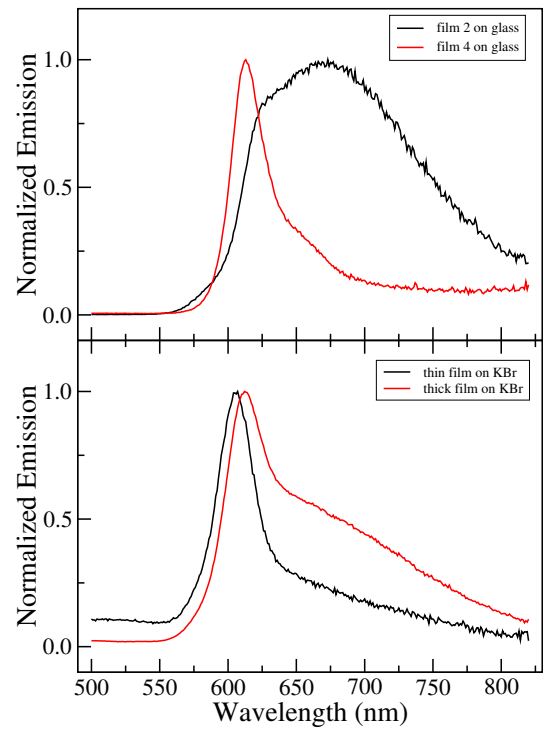

Fig. 11 Luminescence spectra of QA films of glass and on $\mathrm{KBr}$ substrate.

case of pentacene. ${ }^{15}$ Investigations on the relative phase stability and phonon dynamics are currently under way in our laboratory. The present study paves the way to further experimental studies aimed at finding the proper growth conditions for a given polymorph, as well as the corresponding change, hopefully improvement, of device performance.

\section{Conflicts of interest}

There are no conflicts to declare.

\section{Acknowledgements}

The Acknowledgements come at the end of an article after Conflicts of interest and before the Notes and references.

\section{Notes and references}

1 E. D. Głowacki, M. Irimia-Vladu, S. Bauer and N. S. Sariciftci, J. Mater. Chem. B, 2013, 1, 3742-3753.

2 E. D. Głowacki, M. Irimia-Vladu, M. Kaltenbrunner, J. Gsiorowski, M. S. White, U. Monkowius, G. Romanazzi, G. P. Suranna, P. Mastrorilli, T. Sekitani, S. Bauer, T. Someya, L. Torsi and N. S. Sariciftci, Adv. Mater., 2013, 25, 1563-1569.

3 M. Irimia-Vladu, Chem. Soc. Rev., 2014, 43, 588-610.

4 P. Erk, H. Hengelsberg, M. F. Haddow and R. van Gelder, CrystEngComm, 2004, 6, 475-483.

5 T. Salzillo, S. D'Agostino, A. Rivalta, A. Giunchi, A. Brillante, R. G. Della Valle, N. Bedoya-Martínez, E. Zojer, F. Grepioni and E. Venuti, J. Phys. Chem. C, 2018, 122, 18422-18431.

6 C. Binant, B. Guineau and A. Lautié, J. Soc. Dyers Colorists, JSDC, 1990, 106, 187-191.

7 D. S. Filho and C. M. F. Oliveira, J. Mater. Science, 92, 27, 5101-5107.

8 A. D. Squires, R. A. Lewis, A. J. Zaczek and T. M. Korter, J. Phys. Chem. A, 2017, 121, 3423-3429.

9 E. F. Paulus, F. J. J. Leusen and M. U. Schmidt, CrystEngComm, 2007, 9, 131-143. 
10 T. E. Gorelik, C. Czech, S. M. Hammer and M. U. Schmidt, CrystEngComm, 2016, 18, 529-535.

11 A. Brillante, I. Bilotti, R. G. Della Valle, E. Venuti and A. Girlando, CrystEngComm, 2008, 10, 937-946.

12 S. Dunst, E. Karner, M. E. Coppola, G. Trimmel and M. IrimiaVladu, Monats. Chemie - Chem. Monthly, 2017, 148, 863-870.

13 G. Lincke and H.-U. Finzel, Cryst. Res. Techn., 1996, 31, 441452.

14 R. Laudise, C. Kloc, P. Simpkins and T. Siegrist, J. Cryst. Growth, 1998, 187, 449 - 454.

15 A. Brillante, R. D. Valle, L. Farina, A. Girlando, M. Masino and
E. Venuti, Chem. Phys. Lett., 2002, 357, 32 - 36.

16 R. G. Della Valle, E. Venuti, T. Salzillo, A. Brillante, M. Masino and A. Girlando, Int. J. Quantum Chem., 2018, 118, e25503.

17 G. Turrell, Infrared and Raman Spectra of Crystals, Academic Press, London and New York, 1972.

18 S. De Feyter, A. Gesquière, F. C. De Schryver, U. Keller and K. Müllen, Chem. Mater., 2002, 14, 989-997.

19 F. H. Chung and R. W. Scott, J. Appl. Cryst., 1971, 4, 506-511.

20 J. Kalinowski, W. Stampor, P. Di Marco and V. Fattori, Chem. Phys., 1994, 182, 341-352. 